\title{
IdeAs
}

Idées d'Amériques

$18 \mid 2021$

Frontières dans les Amériques - Intégration, sécurité et migrations

\section{De travailleurs mexicains sans-papiers à familles centraméricaines demandeuses d'asile : la transformation des flux migratoires à la frontière Mexique-États-Unis, 2005-2020.}

From Undocumented Mexican Workers to Central American Asylum-seeking

Families: the Transformation of Migration Flows at the Mexico-US Border, 2005-2020.

De trabajadores indocumentados mexicanos a familias centroamericanas solicitantes de asilo: la transformación de los flujos migratorios en la frontera México-Estados Unidos, 2005-2020.

\section{Marie-Laure Coubès}

\section{(2) OpenEdition} Journals

Édition électronique

URL : https://journals.openedition.org/ideas/11653

DOI : $10.4000 /$ ideas. 11653

ISSN : 1950-5701

Éditeur

Institut des Amériques

\section{Référence électronique}

Marie-Laure Coubès, « De travailleurs mexicains sans-papiers à familles centraméricaines demandeuses d'asile : la transformation des flux migratoires à la frontière Mexique-États-Unis, 2005-2020. », IdeAs [En ligne], 18 | 2021, mis en ligne le 01 octobre 2021, consulté le 21 octobre 2021 URL : http://journals.openedition.org/ideas/11653; DOI : https://doi.org/10.4000/ideas.11653

Ce document a été généré automatiquement le 21 octobre 2021.

\section{c)}

IdeAs - Idées d'Amériques est mis à disposition selon les termes de la licence Creative Commons Attribution - Pas d'Utilisation Commerciale - Pas de Modification 4.0 International. 


\section{De travailleurs mexicains sans- papiers à familles centraméricaines demandeuses d'asile : la transformation des flux migratoires à la frontière Mexique-États-Unis, 2005-2020.}

From Undocumented Mexican Workers to Central American Asylum-seeking Families: the Transformation of Migration Flows at the Mexico-US Border, 2005-2020.

De trabajadores indocumentados mexicanos a familias centroamericanas solicitantes de asilo: la transformación de los flujos migratorios en la frontera México-Estados Unidos, 2005-2020.

Marie-Laure Coubès

\section{Introduction}

1 Les villes de la frontière Nord du Mexique sont des lieux de grande mobilité, des espaces de transit de divers flux de migrants qui espèrent entrer aux États-Unis. Tout au long du xxe siècle elles ont été témoins de la migration circulaire entre le Mexique et les États-Unis. Néanmoins, le début du xxi ${ }^{e}$ siècle connait des bouleversements dans l'origine des flux, le profil démographique des migrants et les formes de mobilité vers le pays voisin.

2 Ce changement démarre avec la crise économique de 2008 et la baisse des flux de travailleurs mexicains sans-papiers qui s'est prolongée tant en raison de la politique 
migratoire répressive du gouvernement étasunien (Zenteno R. et R. Suro, 2021) qu'en conséquence du vieillissement démographique du Mexique (Massey D. et al., 2014). Cependant, en Amérique centrale cette réduction de la migration n'a pas lieu et on assiste au contraire à une augmentation des mouvements de population. La situation d'insécurité générale, sociale et économique, du Guatemala, du Honduras et du Salvador pousse chaque fois plus de personnes sur les routes de l'exil. Avec ce changement dans l'origine nationale des migrants, la transformation concerne trois dimensions: le profil démographique (adultes puis familles), les motivations du déplacement (d'une migration économique vers une migration de refuge), et les formes de la mobilité (de la clandestinité à la visibilité). Il est toutefois pertinent de se demander si cette transformation de la prépondérance d'un courant migratoire sur l'autre contient aussi des éléments de changements et de continuité pour chacun des deux courants (mexicain et centraméricain).

Cet article s'attache à démontrer cette évolution dans chacune de ses dimensions. La première partie explique la diminution de la migration des travailleurs mexicains sanspapiers, la seconde l'émergence des familles centraméricaines qui fuient leur pays, et la troisième comment la migration clandestine est devenue visible avec les nouvelles formes de mobilité collective. Finalement, la conclusion propose une interprétation des changements et des continuités des deux mouvements migratoires que cette évolution recèle.

4 L'article mobilise diverses sources de données quantitatives des 15 dernières années, principalement l'enquête des migrations à la frontière nord du Mexique, Emif Norte, et les registres des détentions réalisées par la patrouille frontalière américaine (Border Patrol) $)^{1}$. L'Emif Norte interroge les migrants lorsqu'ils arrivent dans les villes frontalières mexicaines; c'est la seule enquête qui permette de connaitre si les migrants disposent de documents migratoires ou sont sans-papiers. Les registres de détention à la frontière sud-ouest des États-Unis présentent quelques caractéristiques des personnes qui sont retenues ou se sont présentées à la patrouille frontalière : la nationalité et la catégorie démographique (adulte voyageant seul, enfant voyageant seul, membre d'une famille).

\section{De Mexicains à Centraméricains : la chute de la migration mexicaine sans-papier}

5 Tout au long du $\mathrm{xx}^{\mathrm{e}}$ siècle, la migration des Mexicains aux États-Unis a été massive, fréquemment circulaire et sans-papier (Alanis F. et R. Alarcon, 2016). Répondant à la demande du marché du travail des États-Unis et renvoyés au moment des crises, les migrants ont joué le rôle de main-d'œuvre flexible dans les secteurs les plus précaires (agriculture, construction, restauration, etc.) (Bustamante J., et al. 1992). Une importante proportion des migrants se sont installés aux États-Unis, où la population mexicaine est passée de 0,7 million en 1970 à 9,2 millions en 2000 (Zenteno R. et R. Suro, 2021), mais en même temps la migration circulaire a continué, avec les déplacements temporaires des migrants mexicains entre les deux pays avec ou sans documents migratoires $^{2}$ (Massey D. et al., 2014). Pour les sans-papiers, le passage de la frontière était un obstacle surmonté en payant un passeur ou en persévérant, après quelques essais infructueux. Dans les années 1990, la militarisation de la frontière près des grandes villes de Ciudad Juarez et Tijuana a poussé les migrants vers les zones désertiques où les conditions topographiques et climatiques ont rendu le passage 
beaucoup plus risqué (Alonso G., 2013) mais n'ont pas freiné la migration. Ainsi pour l'année 2000, la patrouille frontalière étasunienne a détenu 1,6 millions de Mexicains (USCBP 2019b). A partir du XXI ${ }^{e}$ siècle l'intensification d'une politique répressive du gouvernement américain basée sur l'endiguement des flux à la frontière et la détention des migrants sans-papiers à l'intérieur des États-Unis commence à changer la donne.

Les premières années 2000 ne montrent cependant pas d'effets sur les chiffres de la migration de sans-papiers, car un grand appel de main d'œuvre dans le secteur de la construction fait bondir les flux de Mexicains : il faut construire les maisons vendues avec les prêts qui ont formé la bulle immobilière qui éclate en 2007 (Zenteno R. et R. Suro, 2021). À partir de cette date, on assiste à une importante baisse des flux de migrants sans-papiers. L'enquête réalisée dans les villes frontalières décrit bien ce déclin constant depuis cette date.

7 D'après les données de l'Emif (voir graphique 1), 2007 a été l'année avec le plus de déplacements de personnes sans-papiers souhaitant passer aux États-Unis, 683000, pour ensuite baisser jusqu'à 16000 en $2017^{3}$ : cela correspond à une réduction drastique, le flux de 2017 ne représentant que $2 \%$ du flux 10 ans plus tôt.

Le démarrage de la baisse des flux de migrants mexicains sans-papiers est concomitant avec la crise économique de 2007-2008, et on a pu observer, dans un premier temps, une forte corrélation de ce flux avec le taux de chômage des Mexicains aux États-Unis. Cependant, à partir de 2012, on a observé une déconnexion entre ces deux indicateurs : la migration sans-papier a continué de baisser alors que le chômage diminuait aux États-Unis (Coubès M. et L. Calva, 2017). Toutefois, cette chute du nombre de sanspapiers a été compensée, au moins en partie, par l'augmentation des migrants détenteurs de visas de travailleurs temporaires. Ce sont des visas pour des travailleurs peu qualifiés, dans l'agriculture (H2A) ou dans d'autres secteurs (H2B). Ainsi, sur le marché du travail, la relation entre demande de travail étasunienne et offre de travail mexicaine se fait désormais par le biais de ces visas. Entre 2009 et 2019, les visas H2A pour le secteur agricole attribués aux Mexicains représentent plus de $90 \%$ du total des visas H2A accordés, et ont été multipliés par 3,4, passant de 55693 à $188758^{4}$; entre 2014 et 2018 ils ont augmenté de 20 \% par an (Calva L., 2019), et même en 2020, ils ont augmenté de $5 \%$ (197 908) car, considérés comme travailleurs essentiels, la frontière était ouverte pour eux. Les visas H2B ont un plafond défini et leur nombre varie donc peu (72 339 en 2019); si on les ajoute aux premiers ce sont un total de 261097 visas qui sont délivrés en 2019, un chiffre proche de celui des Mexicains qui déclaraient vouloir traverser la frontière sans-papiers en 2010 (235 000). Ainsi, ces visas sont devenus une option pour ceux qui, quelques années auparavant, auraient traversé la frontière clandestinement ${ }^{5}$. 
Graphique 1 Estimation du flux des migrants arrivés dans une ville frontalière mexicaine et se dirigeant vers les États-Unis, total et sans-papiers, en milliers, 2004-2017

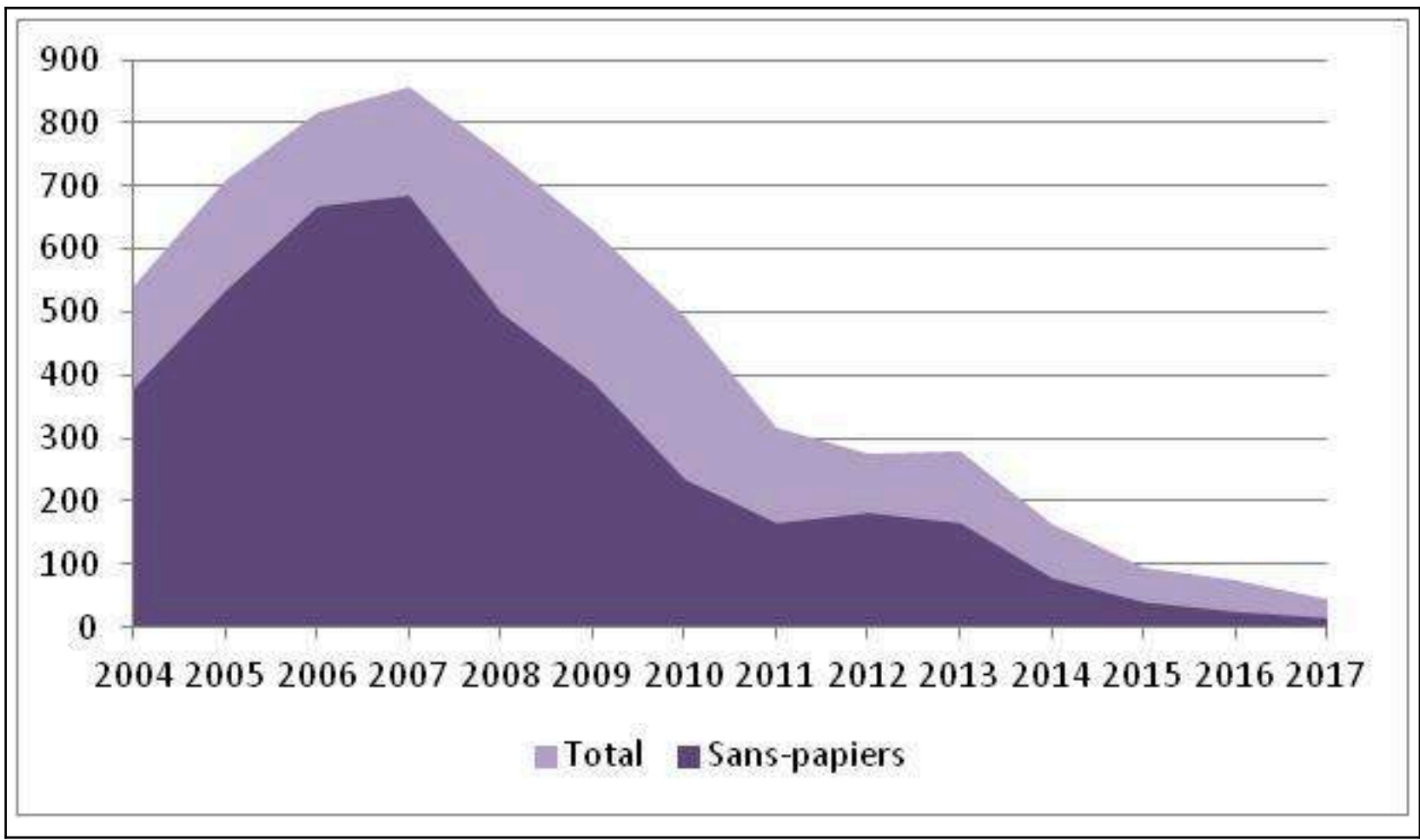

Source : El Colef et al. 2020

Note : Les sans-papiers sont les personnes qui déclarent n'avoir aucun document pour passer la frontière.

9 Si beaucoup préfèrent aller travailler aux États-Unis avec ces visas, alors que la migration circulaire de sans-papiers était traditionnelle dans de nombreuses régions du Mexique, c'est parce que cette dernière est devenue extrêmement difficile et risquée. En effet, bien que des raisons structurelles liées au vieillissement démographique du Mexique expliquent aussi la baisse de la migration mexicaine (Massey D. et al., 2014), la politique de répression du gouvernement étasunien est un facteur essentiel pour expliquer la diminution de la migration de sans-papiers mexicains (Orrenius P. et M. Zavodny, 2014, Zenteno R. et R. Suro, 2021). Le processus de criminalisation de la migration a démarré en 1996 avec la Illegal Immigration Reform and Immigrant Responsibility Act (IIRIRA) votée par le Congrès et s'est développé après 2001 avec la création de l'agence Immigration and Control Enforcement (ICE) en 2003, puis s'est renforcée au moyen de divers programmes «Secure communities" (de 2007 à 2014) et l'opération Streamline de la patrouille frontalière (2008), jusqu'à la politique de "Tolérance zéro" du président Trump (Cornelius W., 2018). Ce processus a pour conséquence que les sanctions pour les entrées se durcissent avec des détentions administratives plus longues et des mises en examen par le système judiciaire pénal fédéral avec emprisonnement à la clef. La catégorie des expulsés par décision judiciaire ( "removed») est désormais la plus importante alors qu'auparavant la majeure partie des personnes détenues à la frontière avaient une expulsion simple («return») sans conséquences légales (Coubès M.-L., 2018) ${ }^{6}$. Par ailleurs, on note une forte augmentation des expulsions des personnes qui résidaient aux États-Unis, avec un pic de 118000 Mexicains en 2011 (Emif Norte). Ces expulsés de l'intérieur sont arrivés en 
nombre dans les villes frontalières, alors que les flux de migrants venus du Sud se tarissaient ${ }^{7}$. Associés à ces expulsions, il y a eu aussi beaucoup de retours pendant la même période. Cependant même les retours (contraints ou forcés) ont diminué, si bien qu'on assiste, dans les cinq dernières années, à une baisse d'intensité de toute la dynamique migratoire entre le Mexique et les États-Unis (Calva L. et M.-L. Coubès 2017).

En conséquence, les détentions de Mexicains à la frontière par les agents de la patrouille frontalière des États-Unis sont en diminution continue depuis 2005 et connaissent une chute libre depuis 2008. De 653035 en 2008 à 166458 en 2019, cela représente une baisse de $75 \%$ des détentions (voir graphique 2). Pendant ce temps, ce sont les détentions de Centraméricains qui ont augmenté à partir de 2012, jusqu'à dépasser celles des Mexicains une première fois en 2014 puis de manière constante depuis 2016 et avec même une augmentation exponentielle en 2019. Le niveau de 2019 est très proche de celui des détentions des Mexicains en 2008. Le graphique des deux courbes représente une forme de vases communicants: dans les registres des détentions à la frontière les personnes centraméricaines ont remplacé les personnes mexicaines.

Graphique 2 : Détentions à la frontière sud-ouest des États-Unis par la patrouille frontalière selon leur nationalité, Mexique et Nord Amérique Centrale (Guatemala, Honduras, El Salvador), 2008-2019 (années fiscales)

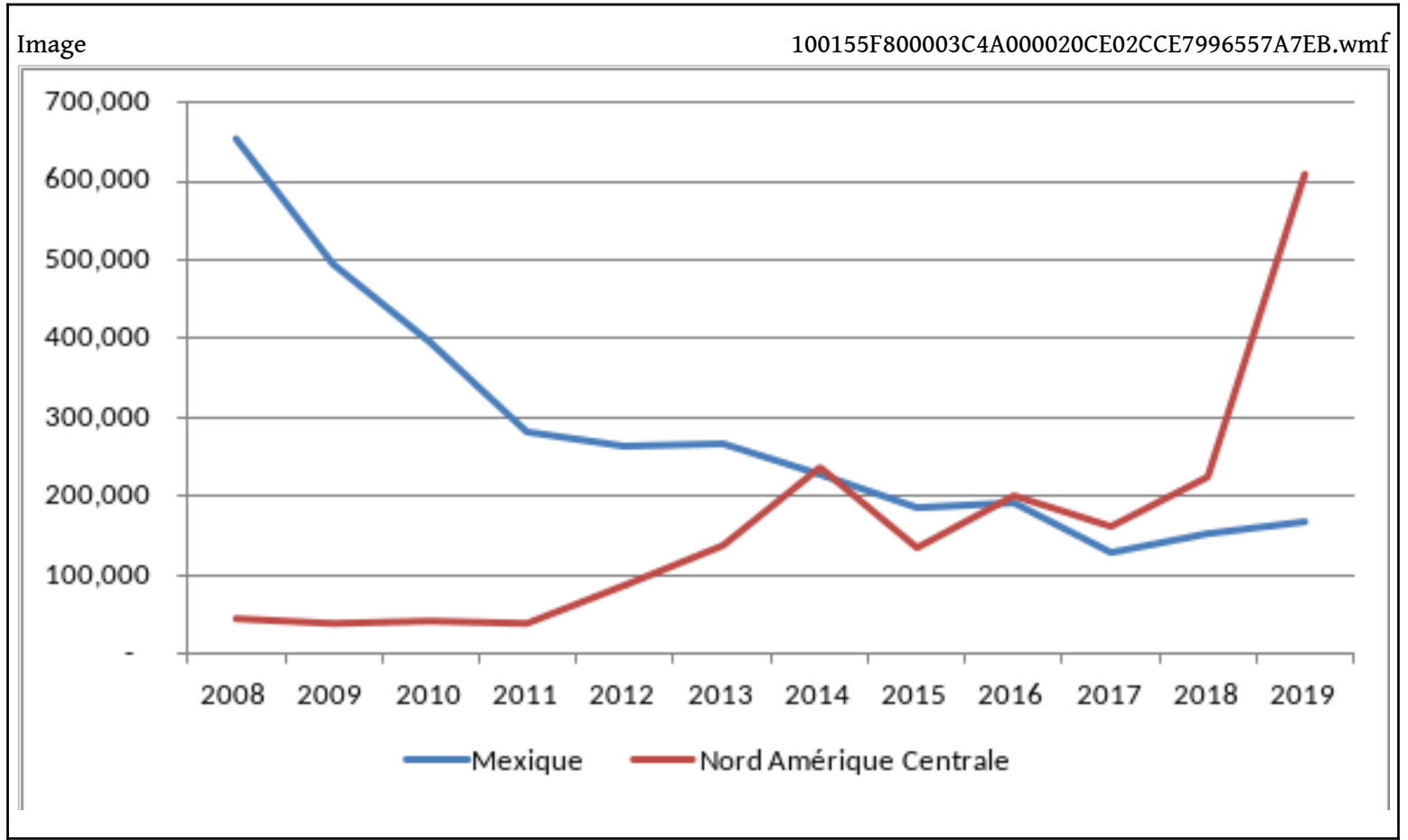

Source : U.S. Customs and Border Protection, 2019b.

Note : les données sont présentées par année fiscale des États-Unis, c'est-à-dire du mois d'octobre d'une année au mois de septembre de la suivante. Par exemple, l'année fiscale 2019 représente la période d'octobre 2018 à septembre 2019. 


\section{De travailleurs à réfugiées : l'exode des familles centraméricaines}

11 La migration centraméricaine est en relation avec l'importante insécurité, économique, sociale et politique, qui prévaut dans les trois pays du nord de l'Amérique centrale (NCA). La pauvreté qui touche les trois-quarts de la population du Honduras, les deuxtiers de celle du Guatemala et des deux-cinquièmes du Salvador, ${ }^{8}$ côtoie des situations de violence et d'insécurité extrême, marquée par le poids des gangs qui délitent la société entière, dans un contexte d'impunité institutionnelle. Indicateurs de la violence, les taux d'homicides sont les plus élevés au monde (hors conflit armé) ${ }^{9}$; et les jeunes en constituent les cibles principales, obligés d'entrer dans les gangs ou victimes d'assassinat. On assiste à un croisement des deux problématiques car l'insécurité affecte l'économie, avec l'extorsion des petits commerçants, les vols des moyens de production et de travail.

Donc, tant les facteurs économiques que les facteurs d'insécurité poussent les Centraméricains sur les routes de l'exil. Cette situation est emblématique des flux mélangés dont parle l'Organisation Internationale des Migrations pour décrire les mouvements de population complexes qui incluent à la fois demandeurs d'asile et migrants économiques (CEPAL 2019 p. 10). Mais ce «brouillage des catégories » (Doraï M., 2008) est aussi présent au niveau micro, car, pour une même personne, il est difficile de séparer et de hiérarchiser les raisons du départ. La situation d'insécurité impacte aussi l'économie, rendant très difficile la survie des petits commerces ou des services indépendants, alors que l'informalité est une caractéristique très forte des marchés du travail des pays du NAC ${ }^{10}$. Ainsi, lorsqu'on leur demande quelles sont les deux principales motivations pour avoir quitté leur pays, la grande majorité des personnes centraméricaines arrivées à la frontière avec les caravanes mentionne une raison liée à la violence des gangs ou à l'insécurité, combinée ou non à des problèmes économiques ou d'emploi ( $68 \%$ dans l'enquête réalisée à Tijuana et $80 \%$ à Piedras Negras) ${ }^{11}$.

13 Dans ce contexte, de très nombreux Centraméricains considèrent qu'ils sont obligés de fuir et se dirigent vers les États-Unis, destination traditionnelle de leur migration. En effet, les EU ont accordé des Statuts de Protection Temporaire (TPS pour son sigle en anglais) aux Salvadoriens qui avaient émigré en masse pendant la guerre civile des années $1980^{12}$ puis après le tremblement de terre de 2001 (TPS prolongé jusqu'à aujourd'hui); et aux Honduriens, depuis 1999, après l'ouragan Mitch de 1998 qui a déclenché leur migration en masse (Pederzini K. et al., 2015). La migration aux ÉtatsUnis fait donc partie de l'histoire des familles du NCA qui ont des réseaux dans ce pays avec un poids économique important ${ }^{13}$. Or ces réseaux sociaux jouent un rôle essentiel dans la perpétuation du processus migratoire (Massey et al. 2014, Aragon 2014).

Par-delà le changement d'origine géographique des migrants, la transformation des flux de migration vers les États-Unis correspond donc à une évolution du type de migration, passant de travailleurs répondant à une demande du marché du travail étasunien à des migrants fuyant des conditions de vie déplorables et espérant un statut de protection de la part des EU, même si ce statut n'est pas accessible aux nouveaux arrivants mais seulement aux migrants sans-papiers déjà installés dans le pays. 

comprend de très nombreuses familles : mères (le plus souvent) et / ou pères avec des enfants en bas âge se déplaçant ensemble pour gagner la frontière étasunienne. Depuis le programme des Braceros (avec le Mexique dans les années 1940) ${ }^{14}$, la migration vers les États-Unis s'était fortement masculinisée et concernait des adultes se déplaçant seuls, qui une fois installés, commençaient un processus de réunification familiale. Les déplacements des dernières années de parents avec leurs enfants, marquent donc un changement du modèle migratoire de la région.

Les données sur les familles procèdent de la patrouille frontalière qui introduit la catégorie des "unités familiales» dans ses registres depuis 2012 : l'unité familiale (family unit) est définie comme toute personne qui fait partie d'un groupe formé par une personne mineure et son père ou mère ou tuteur légal (USDHS, 2019). L'apparition de cette nouvelle catégorie laisse supposer qu'auparavant les détentions de familles n'étaient pas suffisamment nombreuses pour attirer l'attention de cette corporation. En effet, en 2012 et 2013 les personnes se déplaçant en familles ne représentaient que $3 \%$ de toutes les détentions (USDHS, 2019). Or, en 2014 on observe un bond de ces détentions qui représentent $14 \%$ du total (voir graphique 3). Pourtant, ce surcroit, en 2014, a été plutôt occulté par le phénomène des mineurs centraméricains "non accompagnés ", voyageant sans leurs parents, qui arrivaient à la frontière pour solliciter une protection. En même nombre que les membres de familles, 68541 mineurs non accompagnés ont été enregistrés auprès de la patrouille frontalière (USDHS, 2019) ${ }^{15}$. Ils se présentent d'eux même immédiatement aux autorités étasuniennes pour solliciter le Special Immigrant Juvenile Visa prévu pour les immigrants de moins de 21 ans qui ont été abandonnés ou ont souffert d'abus ou de négligence de la part de leurs parents (Rodriguez E., 2016). Les passeurs eux-mêmes ont «vendu » cette option aux familles dans les pays d'origine, et ont convaincu ainsi les parents de laisser partir leurs enfants dans ces conditions ${ }^{16}$.

17 A partir de cette date de 2014, alors que les enregistrements à la frontière de mineurs non accompagnés continuent de représenter entre $12 \%$ et $14 \%$ du total des détentions de la patrouille frontalière, les enregistrements de personnes se déplaçant en famille sont en nombre chaque fois plus important. Il s'agit d'une croissance soutenue de 2014 à 2018, de 68445 à 107 212, puis exponentielle en 2019 puisque le total des détentions de familles atteint 473682 , soit $55,6 \%$ du total (voir graphique 3 ) $^{17}$. En contraste, au cours des sept dernières années, pour les détentions à la frontière, on est passé de $90 \%$ d'adultes voyageant seuls à seulement $35 \%$. Ces familles sont essentiellement centraméricaines (originaire à plus de $90 \%$ du Guatemala, Honduras et El Salvador). En 2019, la majeure partie du flux venu de ces trois pays et enregistré à la frontière était donc composé de personnes se déplaçant en famille, c'est à dire d'adultes accompagnés d'enfants mineurs (par exemple $75 \%$ pour le flux du Honduras). 
Graphique 3 : distribution des détentions à la frontière selon catégorie démographique, 2012-2019 (années fiscales)

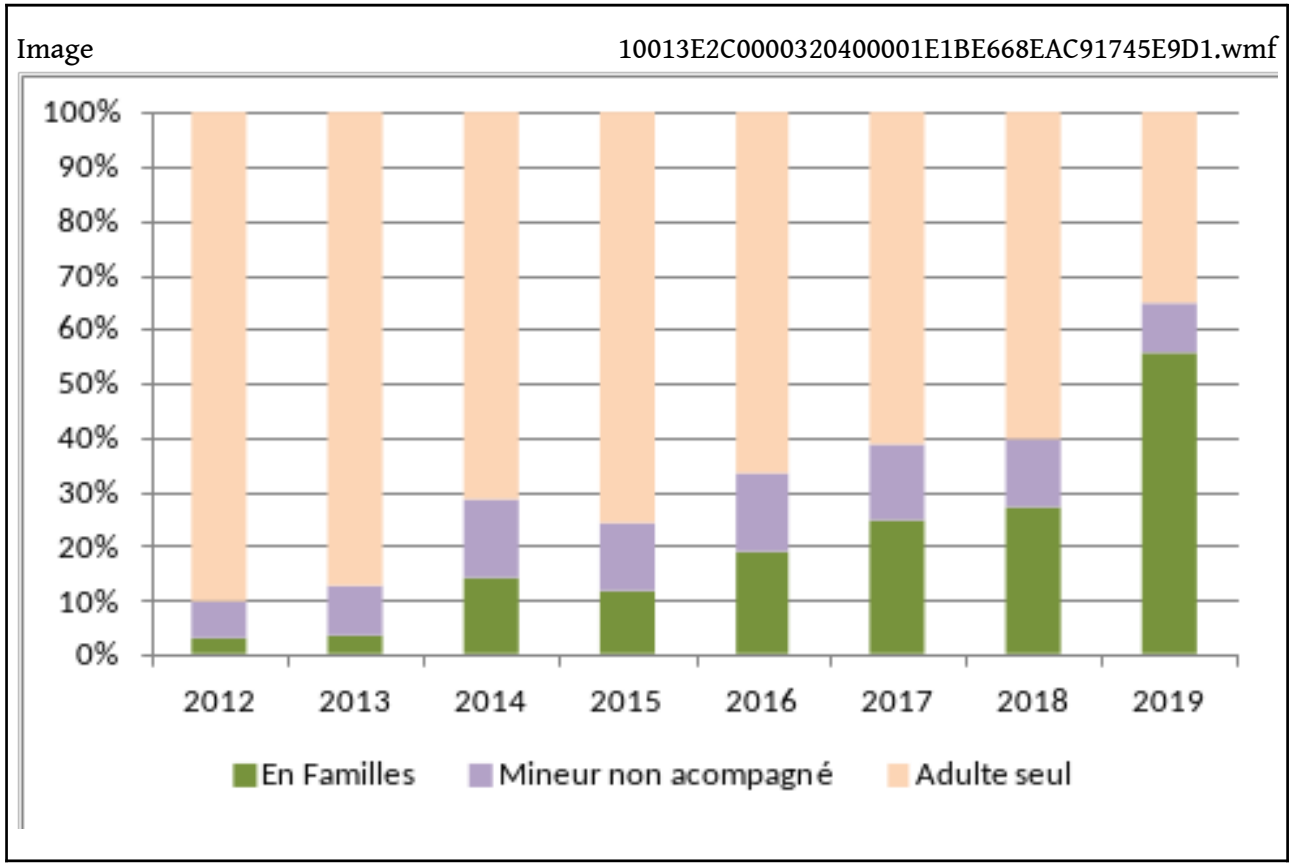

Source : Calculs de l'auteure à partir des données du U.S. Department of Homeland Security (2019) de 2012 à 2017, et du U.S. Customs and Border Protection (2019a), pour 2018 et 2019.

Les déplacements en famille représentent un phénomène récent, objet de nouvelles recherches encore en cours. Une des hypothèses est que les jeunes parents souhaitent maintenir l'unité familiale dans la mobilité, plutôt que d'imposer une séparation, futelle suivie de réunification familiale. Mais certains auteurs soulignent l'aspect instrumental de ce mode de mobilité, les adultes espérant ainsi obtenir plus facilement l'asile s'ils se présentent avec leurs enfants (Martinez R., 2020).

Pour demander l'asile, les migrants se présentent d'eux même aux agents de la patrouille frontalière et cette démarche les rend donc visibles.

\section{De la clandestinité à la visibilité : réfugiés et caravanes}

La clandestinité pour déjouer le contrôle frontalier est une constante de la migration des sans-papiers. La stratégie des travailleurs migrants sans-papiers consistait à entrer clandestinement aux États-Unis pour rejoindre les lieux de travail et se fondre dans la masse des travailleurs et de la population. Relativement facile durant le $\mathrm{xx}^{\mathrm{e}}$ siècle, le passage de la frontière s'est durci à partir des années 1990 pour devenir de plus en plus dangereux et onéreux (Alonso G., 2013). Cependant si la clandestinité reste une ressource elle accroit la vulnérabilité et les risques encourus par les migrants : «Cette économie politique du franchissement clandestin de la frontière découle de la régulation de la migration et de la sécurité frontalière, en liaison avec le modus operandi des cartels de la drogue et du trafic d'êtres humains » (Velasco L., 2018 p. 69). 
21 Pour les Centraméricains, la clandestinité démarre avec la traversée du Mexique. Alors que les États-Unis ont externalisé leur contrôle frontalier c'est l'Etat mexicain qui contrôle le passage de Centraméricains à l'intérieur du pays, qui représente ainsi pour ces derniers une longue frontière 'verticale'18 (Aragon A., 2014, Fitzgerald D., et A. Palomo-Contreras, 2018). Tout au long du trajet, la clandestinité pour éviter la détention et l'expulsion place les migrants en situation de danger face au crime organisé (Paris D., 2018). Accidents, extorsions, enlèvements, viols, assassinats ont rythmé cette route macabre (Aragon A., 2014, Ruiz O., 2001).

22 Ce système de migration clandestine semble s'être épuisé après les assassinats de masse de migrants livrés au crime organisé ${ }^{19}$. Et, depuis octobre 2018, des migrants organisés en caravanes ont pris le parti d'une stratégie inverse : le nombre et la visibilité. La visibilité est une protection pour la traversée du Mexique et un moyen pour solliciter l'asile: à peine entrés sur le territoire étasunien ils recherchent les agents de la patrouille frontalière, et certains se présentent directement aux postes frontaliers ${ }^{20}$.

Différentes actions ont accompagné ce mouvement vers la visibilité de la migration centraméricaine. Les premières caravanes ont été celles de mères de migrants disparus, organisées chaque année au Mexique depuis 2005, pour rendre visible le drame de la migration et les dangers de la traversée du Mexique. Puis, de 2011 à 2015, ce sont les Chemins de croix (Vía crucis), marches-manifestations de migrants au carrefour du politique et religieux, qui se sont développés (Vargas F., 2018).

Empruntant son nom à la caravane, la nouvelle stratégie s'auto-désigne comme un exode de migrants. Le nombre est une protection et il permet un accompagnement exempt des coûts de passeurs pour arriver jusqu'à la frontière des États-Unis sans être cibles des détentions de l'Institut National de Migration, ni victimes du crime organisé (Contreras C. et al., 2021).

25 La visibilité est importante dès le début du parcours de la première caravane, partie le 13 octobre 2018 de San Pedro Sula, au Honduras, avec 1300 personnes réunies par le biais d'une annonce sur une page Facebook. Télévisions et réseaux sociaux diffusent les informations concernant cette caravane et son passage en force de la frontière mexicaine, le 18 octobre. Des municipalités mexicaines mettent à disposition des lieux pour dormir et se restaurer et surtout des moyens de transport pour que la caravane continue sa route (et ne s'arrête donc pas chez eux). Tout au long du trajet, des personnes se joignent à elle, notamment des migrants se trouvant déjà dans le sud du Mexique. Dès le lendemain du départ, ils étaient 2000 puis 4000 à la frontière du Mexique. Trois autres caravanes sont parties de San Pedro Sula et San Salvador entre le 20 et 31 octobre, et plus de 6000 personnes sont arrivées à Tijuana entre le 10 et 27 novembre (El Colef, 2019 p.48).

Les caravanes ne représentent qu'une petite partie des arrivées. Pourtant, cette nouvelle forme de mobilité collective a généré un impact médiatique et politique encore plus important que les flux clandestins. La réponse du gouvernement des ÉtatsUnis est de deux ordres : d'une part, des mesures symboliques ${ }^{21}$ pour faire obstacle aux essais de passage de la frontière, en la fermant quelques heures à San Diego en novembre 2018, ou en doublant les effectifs de la patrouille à Eagle Pass en février 2019 (Contreras C. et al., 2021); d'autre part, le développement de politiques visant à empêcher ou à décourager les ressortissants des trois pays de solliciter l'asile. Parmi ces mesures, dès juin 2018, le Fiscal General, Jeff Sessions, avait annoncé que seule la violence "publique» exercée par un gouvernement contre ses citoyens était 
susceptible d'être retenue comme raison légitime pour obtenir l'asile, écartant de ce fait les violences dues à une "activité criminelle privée" (telle que la violence domestique, violence de gangs, etc.). De même, la politique de séparation des familles de mai 2018, dont l'application a été suspendue le mois suivant dû aux fortes pressions de la société civile étasunienne, visait à décourager les familles. Puis, en 2019, est établi le Protocole de Protection des Migrants (MPP en anglais). Ce dernier consiste à renvoyer les demandeurs d'asile au Mexique pour la durée de l'étude de leur requête aux États-Unis. De mars 2019 jusqu'à janvier 2021, 71039 personnes ont été renvoyées au Mexique dans le cadre de ce dispositif (Université de Syracuse, 2021).

Alors que la pandémie du Covid-19 avait mis un coup d'arrêt à la mobilité avec la fermeture des frontières, les mouvements migratoires ont repris dès la fin de 2020, bien que la frontière terrestre des États-Unis soit toujours fermée au trafic non essentiel depuis le 21 mars 2020. La patrouille frontalière donne le chiffre de 533743 Centraméricains pour l'année fiscale 2021 en cours (octobre à juillet) ${ }^{22}$, proche du maximum de 2019, avec $36 \%$ de familles; si cette proportion est moindre qu'en 2019, elle reste cependant élevée. La mobilité collective en caravanes ayant démontré son efficacité, au moins pour arriver jusqu'à la frontière nord du Mexique, les caravanes de familles ont resurgi alors que la pandémie Covid-19 n'est pas encore dépassée ${ }^{23}$.

La frontière terrestre des États-Unis a été fermée, sauf pour activités essentielles, dans lesquelles les demandes d'asile ne sont pas inclues. Ainsi les listes d'attente sont closes et les rendez-vous dans les tribunaux de migration du département de la Justice sont suspendus pour tous les Centraméricains bloqués du côté mexicain de la frontière. Alors que le protocole MPP avait été annulé par le nouveau président Biden en février 2021, celui-ci a été réimposé par la Cour Suprême en août 2021.

Avec leur statut très précaire au Mexique (une autorisation d'entrée sans droit à travailler), les migrants centraméricains bloqués aux portes des États-Unis sont « attrapés » dans cette immobilité. Ce concept « d'attrapement » développé par Nuñez G. et J. Heyman (2007) pour les migrants dans les villes frontalières étasuniennes peut s'appliquer désormais à la situation actuelle des Centraméricains dans les villes frontalières mexicaines.

\section{Conclusion}

Dans le flux principal de migrants arrivés à la frontière étasunienne, les familles centraméricaines réfugiées ont désormais remplacé les travailleurs sans-papiers mexicains. Ce passage d'une migration d'adultes mexicains à une migration de familles centraméricaines constitue une transformation qui comprend à la fois le profil démographique des migrants, les motivations de la mobilité et les conditions du déplacement. Cependant, ce bouleversement du panorama migratoire des villes frontalières représente pour chaque courant migratoire (mexicain et centraméricain) une évolution avec sa part de changements et de continuités.

31 Dans un contexte démographique de moindre pression migratoire qui limite le nombre de candidats au départ, les Mexicains se sont orientés vers les visas de travail temporaire (accordés de façon croissante par le gouvernement étasunien) pour éviter les coûts de la répression de la circulation sans-papier. Cette migration répond toujours à la demande du marché du travail étasunien car l'économie américaine est la grande force qui attire les migrants mexicains. Dans l'histoire plus que centenaire de cette 
migration, se sont succédées périodes où dominaient la migration régulière puis la migration sans-papier; ainsi, la transformation actuelle (diminution du nombre de sans-papiers) peut se lire comme une nouvelle variation dans le modèle traditionnel de l'émigration de travail.

De la même façon, on peut appréhender l'émigration massive de familles centraméricaines actuelle comme une transformation dans la continuité de l'histoire migratoire de ces pays d'Amérique centrale. Contrairement aux Mexicains, les Centraméricains n'ont pas cette tradition de réponse à la demande du marché du travail étasunien. Et, actuellement, le nombre de visas H2A et H2B accordés aux pays d'Amérique centrale est sans commune mesure avec ceux accordés aux Mexicains (même en tenant compte des rapports de populations entre pays). L'histoire migratoire des Centraméricains des quatre dernières décennies s'est forgée autour de catastrophes sociales (guerres civiles) ou naturelles (cyclones, tremblements de terre) et de la recherche d'un refuge aux États-Unis. Les flux actuels qui sollicitent l'asile aux ÉtatsUnis s'inscrivent dans cette "tradition» de fuite et de demande de protection. La nouveauté est l'importance des familles et la visibilité du déplacement.

Cette étude a donc démontré que la transformation des flux migratoires joue à deux niveaux, le premier est la prépondérance numérique d'un courant migratoire sur l'autre (centraméricain vs mexicain) et le second la modification du mode migratoire de chaque courant alors que se maintient constante la motivation de la migration pour chacun d'eux.

Dans la dernière décennie, la réponse du gouvernement étasunien aux nouveaux flux centraméricains a été une fin de non recevoir avec l'imposition du retour des demandeurs d'asile au Mexique pendant le traitement de leur demande, rejetée pour la grande majorité. Cette politique a des répercussions importantes pour les villes frontalières mexicaines qui sont devenues les lieux de l'attente et de l'immobilité forcée des familles centraméricaines, arrivées en masse ces dernières années. Leur situation juridique de grande vulnérabilité sans droit au travail, crée des situations de précarité structurelle, sans avenir clair, car malgré le peu de succès de leurs demandes d'asile aux États-Unis, seule une minorité des Centraméricains envisagent de s'installer au Mexique et de s'intégrer à la population locale (Velasco L. et al. 2021).

\section{BIBLIOGRAPHIE}

Alanís Enciso, Fernando Saúl et Rafael Alarcón Acosta (dir.), El ir y venir de los norteños. Historia de la migración mexicana a Estados Unidos (siglos XIX-XXI), Tijuana, El Colegio de la Frontera Norte, El Colegio de San Luis, El Colegio de Michoacán, 2016.

Alonso Meneses, Guillermo, El desierto de los sueños rotos. Detenciones y muertes de migrantes en la frontera México-Estados Unidos 1993-2013, Tijuana, El Colegio de la Frontera Norte, 2015.

Aragón, Argán, Migrations clandestines d'Amérique centrale vers les États-Unis, Paris, Presses Sorbonne Nouvelle, 2014. https://books.openedition.org/psn/109 page consultée le 3 mai 2021 
Bustamante, Jorge A., Clark Winton Reynolds et Raúl Andrés Hinojosa Ojeda (dir.), U.S.-Mexico Relations. Labor Market Interdependence, Stanford, Stanford University Press, 1992.

Calva Sánchez, Luis Enrique, «El sistema de visas H2 a inicios de la administración del presidente Donald Trump », Coyuntura Demográfica, n 15, enero 2019, p. 77-83. http:// coyunturademografica.somede.org/wp-content/plugins/coyuntura_demografica/ DEMOGRAFICA/ARTICULOS/PUB-2019-15-175.pdf page consultée le 3 mai 2021

Calva, Luis Enrique et Coubès, Marie-Laure « Desaceleración de la dinámica migratoria : descenso generalizado de los flujos de salida y de retorno de migrantes mexicanos », La situación demográfica de México 2016, 19 de junio de 2017, p. 209-219. https://www.gob.mx/cms/uploads/ attachment/file/232083/09_Calva_Coubes.pdf, page consultée le 3 mai 2021.

Comisión Económica para América Latina y el Caribe (CEPAL), Observatorio Demográfico 2018, (LC/ PUB.2018/25-P), Santiago, 2019.

Comisión Económica para América Latina y el Caribe (CEPAL), CEPALSTAT, [en ligne], Santiago, 2020. https://estadisticas.cepal.org/cepalstat/Portada.html page consultée le 3 mai 2021.

Consejo Nacional de Población et El Colegio de la frontera norte, Prontuario sobre movilidad y migración internacional en la frontera sur de México, México, Conapo, El Colef, 2018. https:// www.gob.mx/conapo/documentos/prontuario-sobre-movilidad-y-migracion-internacional-enla-frontera-sur-de-mexico page consultée le 3 mai 2021

Contreras Delgado, Camilo, María Dolores París Pombo et Laura Velasco Ortiz (dir.) Caravanas migrantes y desplazamientos colectivos en la frontera México-Estados Unidos, Tijuana, El Colegio de la frontera norte, 2021.

Cornelius, Wayne A., « Constructing Deterrence in the Age of Trump: Restricting Asylum, Separating Families, and Criminalizing Migration », LASA Forum, 49:4, October 2018, p. 37-41.

Coubès, Marie-Laure, « Deportaciones de mexicanos desde Estados Unidos: ¿ qué está cambiando con el nuevo gobierno de Estados Unidos ? », Coyuntura Demográfica,n ${ }^{\circ}$ 13, enero 2018, p. 87-95. http://coyunturademografica.somede.org/wp-content/plugins/coyuntura_demografica/ DEMOGRAFICA/ARTICULOS/PUB-2018-13-152.pdf, page consultée le 13 octobre 2021

Calva, Luis Enrique et Coubès, Marie-Laure, « Recent trends in migration: in and out of the Northern and Southern Mexican borders », PAA 2017 Annual Meeting, Chicago, 28 Avril 2017.

Doraï, Mohamed Kamel, « Du brouillage des catégories de réfugié et de demandeur d'asile à partir d'exemples moyen-orientaux ", in Luc Cambrézy, Smaïn Laacher, Véronique Lassailly-Jacob, Luc Legoux (Ed.) L'asile au Sud, 2008, p. 89-109.

El Colegio de la Frontera Norte (El Colef), La caravana de migrantes centroamericanos en Tijuana 2018-2019 (segunda etapa), rapport de recherche, Tijuana, El Colegio de la Frontera Norte, 2019, https://www.colef.mx/wp-content/uploads/2019/03/2o.-Reporte-Caravana-Tijuana.250319.pdf, page consultée le 3 mai 2021.

El Colegio de la Frontera Norte, Unidad de Política Migratoria, Consejo Nacional de Población, Consejo Nacional para Prevenir la Discriminación, Secretaría del Trabajo y Previsión Social, Secretaría de Relaciones Exteriores, et Secretaría de Bienestar (BIENESTAR), Encuesta sobre Migración en la Frontera Norte de México, Serie histórica. https://www.colef.mx/emif/tabulados.html, page consultée le 3 mai 2021.

Fitzgerald, David et Areli Palomo-Contreras, « Le Mexique entre Sud et Nord », in Florence Boyer, Françoise Lestage et María-Dolores París Pombo (dir.), Routes et pauses des parcours migratoires : Afrique-Amérique, Cahiers CEMCA Série Anthropologie, Numéro 03, Novembre 2018 p. 16-32. 
Gilman, Denise, « Barricading the Border: COVID-19 and the Exclusion of Asylum Seekers at the U.S. Southern Border » Frontier Human Dynamics, 2:595814, 2020. Doi: 10.3389/fhumd.2020.595814

Lesnes Corine «A Nogales, l'entrepôt à enfants clandestins », Le Monde, 19 juin 2014, https:// www.lemonde.fr/etats-unis/article/2014/06/19/a-nogales-l-entrepot-a-enfantsclandestins_5992136_1666848.html, page consultée le 29 octobre 2020.

Martínez Casas, Regina, « ¿ De qué tamaño es la actual crisis migratoria en México ? », LASA Forum, 50:4, october 2019, p. 61-63.

Massey, Douglas, Durand, Jorge et Pren, Karen « Explaining Undocumented Migration to the U.S. » The International Migration Review, Winter 2014, vol. 48, nº 4 (Winter 2014), p. 1028-1061.

Núñez, Guillermina Gina et Josiah McC. Heyman, « Entrapment Processes and Immigrant Communities in a Time of Heightened Border Vigilance », Human Organization, vol. 66, $\mathrm{n}^{\circ} 4$ (Winter 2007), p. 354-365.

Orrenius, Pia et Madeline Zavodny, « Unauthorized Mexican Workers in the United States: Recent Inflows and Possible Future Scenarios », Working Paper 436. Washington, DC : Center for Global Development, 2016, http://www.cgdev.org/publication/unauthorized-mexican-workers-unitedstates-inflows, page consultée le 3 mai 2021.

París Pombo, María-Dolores, «Le Mexique, pays de transit ou pierre d'achoppement des migrants centraméricains en route vers les États-Unis ? » in Boyer, Florence, Lestage, Françoise et París Pombo, María-Dolores (dir.), Routes et pauses des parcours migratoires : Afrique-Amérique, Cahiers CEMCA Série Anthropologie, n 3, novembre 2018 p. 41-50. https://cemca.org.mx/es/rutas-ypausas-de-los-recorridos-migratorios-africa-america/, page consultée le 3 mai 2021

Pederzini, Carla, Riosmena, Fernando, Masferrer, Claudia, et al., « Tres décadas de migración desde el triángulo norte centroamericano : Un panorama histórico y demográfico », CANAMID Policy Brief Series, PB01, CIESAS : Guadalajara, México, octubre 2015, http://www.canamid.org/ publication?id=PB01, page consultée le 3 mai 2021.

Reina, Elena, «La pandemia no frena a una nueva caravana de migrantes centroamericanos », $E l$ País, México, 2 octobre 2020 https://elpais.com/mexico/2020-10-01/la-pandemia-no-frena-auna-nueva-caravana-de-migrantes-centroamericanos.html, page consultée le 3 mai 2021.

Rodriguez, Ernesto, Central American Migrants in Irregular Transit through Mexico: news figures and trends. CANAMID Policy Brief Series, PB14, CIESAS, Guadalajara, México, 2016. http:// www.canamid.org/en/publication?id=PB014, page consultée le 30 août 2021

Ruiz, Olivia Marrujo, « Los riesgos de cruzar. La migración centroamericana en la frontera México-Guatemala », Frontera Norte, n 13 (25), 2001, p. 7-33. http://www.scielo.org.mx/ scielo.php?script=sci_arttext\&pid=S0187-73722001000100001, page consultée le 13 octobre 2021.

U.S. Department Of State, Bureau Of Consular Affairs. Nonimmigrant Visa Issuances by Visa Class and by Nationality: https://travel.state.gov/content/travel/en/legal/visa-law0/visa-statistics/ nonimmigrant-visa-statistics.html page consultée le 30 août 2021

U. S. Customs and Border Protection (CBP), U. S. Border Patrol Southwest Border Apprehensions by Sector Fiscal Year 2019, [en ligne], November 14 2019, 2019a https://www.cbp.gov/newsroom/ stats/sw-border-migration/usbp-sw-border-apprehensions-fy2019, page consultée le 3 mai 2021.

U. S. Customs and Border Protection (CBP), U.S. Border Patrol Apprehensions From Mexico and Other Than Mexico (FY 2000 - FY 2019), [en ligne], November 14 2019, 2019b. https://www.cbp.gov/ document/stats/us-border-patrol-apprehensions-mexico-and-other-mexico-fy-2000-fy-2019 , page consultée le 3 mai 2021. 
U. S. Department of Homeland Security (DHS), DHS Border Security Metrics Report, [en ligne], February 26 2019, https://www.dhs.gov/sites/default/files/publications/ ndaa_border_metrics_report_fy_2018_0_0.pdf, page consultée le 3 mai 2021.

Université de Syracuse, Details on MPP (Remain in Mexico) Deportation Proceedings, $2021 \mathrm{https://}$ trac.syr.edu/phptools/immigration/mpp/page consultée le 3 mai 2021.

Vargas Carrazco, de Jesús, Felipe « El vía crucis del migrante : Demandas, membresía e interlocutores ", Trace, 73, enero 2018, p. 117-133. DOI: http://dx.doi.org/10.22134/trace. 73.2018 .88

Valenzuela Arce, Manuel, Jose, Naterras, Alfredo et al. (coord.) Las Maras. Identidades juveniles al límite, Tijuana, El Colegio de la Frontera norte, Juan Pablos Editores, Universidad Autónoma Metropolitana, 2007.

Velasco Ortiz, Laura, « Frontières, mobilité et clandestinité dans la région Mexique-États-Unis », in Florence Boyer, Françoise Lestage et María-Dolores París Pombo (dir.), Routes et pauses des parcours migratoires: Afrique-Amérique, Cahiers CEMCA Série Anthropologie, $\mathrm{n}^{\circ} 3$, novembre 2018 p. 61-70. https://cemca.org.mx/es/rutas-y-pausas-de-los-recorridos-migratorios-africaamerica/, page consultée le 13 octobre 2021.

Velasco Ortiz, Laura (Coord.), Entre la espera y el asentamiento: inserción laboral y residencial de inmigrantes y desplazados en ciudades fronterizas del norte de México : los casos de Tijuana y Ciudad Juárez, Reporte Ejecutivo, El Colegio de la Frontera Norte, UCLA. 2021. https://www.colef.mx/ estudiosdeelcolef/entre-la-espera-y-el-asentamiento-insercion-laboral-y-residencial-deinmigrantes-y-desplazados-en-ciudades-fronterizas-del-norte-de-mexico/, page consultée le 30 août 2021.

Zenteno, René et Suro, Roberto « Recession Versus Removals, Which Finished Mexican Unauthorized Migration? », in Hinojosa, Raul et Telles, Edward (dir.), The Trump Paradox: Migration, Trade, and Racial Politics in US-Mexico, Berkeley, California, University of California Press, 2021.

\section{NOTES}

1. L'Emif Norte, étudie depuis 1993 les flux migratoires mexicains dans les villes frontalières mexicaines. Basée sur l'observation continue des différents flux (procédant du Sud ou du Nord), l'enquête applique des techniques de sondage probabiliste des populations mobiles. Elle a été créée par des chercheurs du El Colegio de la Frontera Norte, centre public de recherche du Mexique, et est financée par divers ministères mexicains. (cf. le site Internet, en espagnol, www.colef.mx/ emif).

Les données des registres administratifs des détentions réalisées à la frontière sud-ouest des États-Unis (frontière terrestre avec le Mexique) sont publiées pour chaque année sur le site web de l'United States Customs and Border Protection (USCBP).

2. La loi Immigration Reform and Control Act (IRCA) de 1986 a permis que 2266577 Mexicains sanspapiers obtiennent leur régularisation dans les 5 années suivantes (Alanis F. et R. Alarcon, 2016, p.310).

3. Pour des raisons budgétaires, en 2018 et 2019 l'enquête n'a pas été réalisée pour ce flux particulier.

4. Calculs de l'auteure à partir des données du U.S. Department of States, Bureau of Consular Affairs

5. Ces travailleurs avec visa sont peu représentés dans l'enquête Emif car beaucoup prennent directement un vol international depuis le centre du Mexique. 
6. Les migrants qui sont expulsés selon la catégorie de "removed" ont une interdiction de séjour de plusieurs années (voire de toute la vie) avant de pouvoir solliciter de nouveau une entrée aux États-Unis. La catégorie des "return" consiste en signer une déclaration de "retour volontaire" sans conséquence administrative ni légale. La politique de tolérance zéro détermine que toute personne entrée illégalement serait poursuivie légalement, ce qui a généré la séparation des familles dont nous parlerons dans la dernière section. Cette politique a été annulée par le gouvernement Biden en janvier 2021.

7. Particulièrement à Tijuana où la proximité de la métropole de Los Angeles permet aux personnes expulsées de rester proches de leur famille qui pourront venir leur rendre visite.

8. Consejo Nacional de Población (Conapo) et El Colegio de la Frontera norte (Colef), 2018, p. 20.

9. Au classement mondial de 2015, le Salvador est le premier pays (109 homicides pour 100000 habitants), le Honduras le deuxième (64 pour 100000$)$ et le Guatemala le septième ( 31 pour 100 000). Conapo et El Colef, 2018, op.cit., p. 21.

10. Selon CEPALSTAT en 2018 le secteur informel représente $58 \%$ du marché du travail urbain au Honduras, $57 \%$ au Guatemala et $54 \%$ au Salvador (données de 2014 pour le Guatemala) (CEPAL 2020).

11. Calculs de l'auteur basés sur les enquêtes Condiciones de Movilidad y Estancia de la Población Integrante de la Caravana Migrante. El Colegio de la frontera norte, Tijuana décembre 2018 et Piedras Negras février 2019. Nous présentons les caravanes de migrants dans la section suivante.

12. Premier TPS entre 1990 et 1992 prolongé jusqu'en 1995 avec Deferred Enforcement Departure. Ce statut correspond à une protection limitée puisqu'il ne donne pas droit à la résidence permanente.

13. Dans les flux récents, $98 \%$ des salvadoriens en transit au Mexique pour les États-Unis déclarent avoir de la famille dans ce pays, $88 \%$ du Honduras et $83 \%$ du Guatemala. Conapo et Colef, 2018, op.cit., p. 14-16 et 47. L'apport de la migration à l'économie des familles s'observe au niveau national avec les réceptions d'argent qui représentent une forte proportion du PIB de chaque pays : $18 \%$ Salvador, $17 \%$ Honduras et $11 \%$ Guatemala. Conapo et Colef, 2018, op.cit., p. 23.

De plus, les interactions entre migration et violence sont très complexes puisque ce sont les expulsions depuis les États-Unis, dans les années 90, des jeunes membres de gangs, qui ont exporté et transnationalisé les bandes telles que Mara Salvatrucha et Barrio 18, pour les plus connues, dans ces pays. (Valenzuela J. et al. 2007)

14. Programme de travail temporaire pour répondre aux besoins de main d'œuvre alors que les jeunes étasuniens sont partis au front de guerre (Alanis F. et R. Alarcon, 2016)

15. Leur réception dans des centres grillagés avait fait les premières pages des médias internationaux (Lesnes C., 2014).

16. Communication personnelle avec M. Arevalo, chercheur de la FLACSO Guatemala.

17. Rappelons que les détentions représentent des évènements car les personnes peuvent être enregistrées plusieurs fois la même année.

18. Souvent appelée frontière verticale en opposition à la «frontière horizontale » de la ligne de démarcation internationale.

19. Le plus tristement célèbre est le massacre de San Fernando, localité du Tamaulipas où 72 personnes migrantes ont été assassinées par des membres du cartel des Zetas en 2010.

20. On peut estimer à $24 \%$ en 2018 et $13 \%$ en 2019 ceux qui se sont présentés aux postes d'entrée (calculs de l'auteure à partir des données des personnes considérées comme non admissibles par le Bureau des Opérations de terrain, OFO pour son sigle en anglais)

21. Ces mesures sont symboliques car ces villes avaient déjà des murs qui rendent le passage en force pratiquement impossible.

22. Depuis mars 2020, les données de la patrouille frontalière étasunienne incluent les personnes expulsées au titre de la pandémie, en référence au titre 42 du Code des États-Unis. Ces expulsions 
express ne sont pas basées sur le statut migratoire des personnes cependant selon (Gilman D., 2020), elles sont appliquées essentiellement aux demandeurs d'asile.

23. Dès l'annonce de l'ouverture de la frontière entre le Honduras et le Guatemala, en octobre 2020, une nouvelle caravane s'était formée (Reina E., 2020). Ensuite plusieurs autres ont démarré leur route mais ont été stoppées avant même d'arriver au Mexique. La plus récente (août 2021) s'est formée avec des migrants déjà entrés au Mexique mais qui étaient maintenus en attente dans le Sud du pays.

\section{RÉSUMÉS}

Depuis plus de 10 ans on a assisté à une baisse drastique de la migration mexicaine sans-papiers vers les États-Unis, liée à la crise économique de 2008, à la politique répressive des États-Unis ainsi qu'au vieillissement démographique du Mexique. Cependant ce ralentissement ne touche pas les pays du triangle nord d'Amérique centrale où la situation économique et sociale désastreuse pousse des familles sur les routes de l'exil. Ces déplacements de familles s'accompagnent d'une transformation de la mobilité, passée de la clandestinité pour déjouer le contrôle frontalier à une stratégie de visibilité tant pour éviter les dangers de la traversée clandestine du Mexique que pour demander la protection internationale. Cet article mobilise diverses sources de données quantitatives, les registres des détentions à la frontière par les autorités des États-Unis et les enquêtes continues réalisées auprès des migrants aux frontières du Mexique (Emif). Cette perspective socio-démographique d'analyse des évolutions des 15 dernières années démontre que les flux migratoires à la frontière des États-Unis se sont radicalement transformés: les travailleurs mexicains clandestins ont été remplacés par des familles centraméricaines demandeuses d'asile.

Mexican migration to the United States has been dropping drastically in the last 13 years, which is linked to the 2008 economic crisis, the repressive policies of the United States as well as the demographic aging in Mexico. However, this slowdown does not affect countries of the northern triangle of Central America where the dire economic and social situation is pushing families onto the roads of exile. These displacements are accompanied by a transformation of mobility, from clandestine move in order to elude border control to a strategy of visibility both to avoid the dangers of crossing Mexico clandestinely and to seek international protection. In this article we mobilize various sources of quantitative data, the records of border detentions by U.S. authorities and the continuous surveys carried out on migrants at the borders of Mexico (Emif). This socio-demographic perspective of analyzing the evolutions of the last 15 years shows that the migratory flows at the US border have been radically transformed: undocumented Mexican workers have been replaced by Central American asylum-seeking families.

La migración mexicana indocumentada a los Estados Unidos ha experimentado una caída drástica desde hace más de 10 años, en relación con la crisis económica de 2008, la política represiva de Estados Unidos, y el envejecimiento demográfico en México. Sin embargo, esta desaceleración no afecta a los países del triángulo norte de Centroamérica donde la adversa situación económica y social está empujando a las familias a huir de su país. Estos desplazamientos de familias van acompañados de una transformación de la movilidad, desde la clandestinidad para evadir el control fronterizo hacia una estrategia de visibilidad tanto para evitar los peligros del tránsito de 
México como para pedir protección internacional. En este artículo movilizamos diversas fuentes de datos cuantitativos, los registros de detenciones en la frontera por parte de las autoridades de Estados Unidos y las encuestas continuas realizadas a migrantes en las fronteras de México (Emif). Esta perspectiva sociodemográfica de análisis de las evoluciones de los últimos 15 años muestra que los flujos migratorios en la frontera de Estados Unidos se han transformado radicalmente: los trabajadores mexicanos indocumentados han sido reemplazados por familias centroamericanas solicitantes de asilo.

\section{INDEX}

Mots-clés : Flux migratoire, sans-papier, réfugié, frontière Mexique États-Unis, centraméricains Keywords : Migration flow, Undocumented, Refugee, U.S. Mexico Border, Central Americans Palabras claves : Flujo migratorio, indocumentado, refugiado, frontera México-Estados Unidos, centroamericanos

\section{AUTEUR}

\section{MARIE-LAURE COUBÈS}

Démographe, chercheure et enseignante au département d'études de population de El Colegio de la Frontera Norte (COLEF). Ses domaines de recherche incluent l'emploi et la mobilité. De 2010 à 2018 elle a dirigé les enquêtes de migration aux frontières Nord et Sud du Mexique (Emif Norte et Emif Sur).mcoubes@colef.mx. 